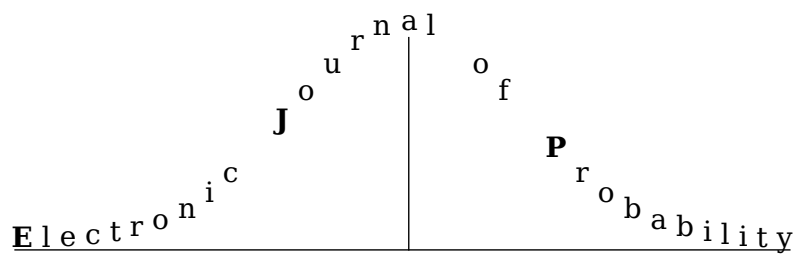

Electron. J. Probab. 26 (2021), article no. 105, 1-17.

ISSN: 1083-6489 https://doi.org/10.1214/21-EJP675

\title{
The maximal difference among expert's opinions
}

\author{
Stanisław Cichomski* $\quad$ Adam Osękowski $^{\dagger}$
}

\begin{abstract}
We prove the sharp bound for the expected spread upon opinions of $n \geq 2$ experts, who have access to different information sources represented by different $\sigma$-fields. Using symmetrization argument and direct combinatorial optimization we derive an explicit optimizer. Our results may turn out to be helpful not only for probabilists, but also for statisticians and economists.
\end{abstract}

Keywords: coherent; opinion; sharp inequality; joint distribution of conditional expectations. MSC2020 subject classifications: $60 \mathrm{E} 15$.

Submitted to EJP on April 23, 2021, final version accepted on July 2, 2021.

\section{Introduction}

The purpose of this paper is to establish a certain sharp maximal estimate for dependent random variables which stems from applications in statistics and information theory. We start with the motivation and the necessary background. Imagine a group of $n \geq 2$ experts with an access to different knowledge, who are asked to evaluate the odds of some future event. The question is: how radically different can their opinions be? This natural and interesting problem can be formalized using the notion of conditional probability. First, our experts must agree upon a basic model of reality, which can be understood as accepting a common probability space $(\Omega, \mathcal{F}, \mathbb{P})$. Inconsistent sources of information shall then be identified with different sub- $\sigma$-fields $\mathcal{G}_{1}, \mathcal{G}_{2}, \ldots, \mathcal{G}_{n} \subset \mathcal{F}$. Consequently, opinions on an event $A \in \mathcal{F}$ will be expressed as random variables $X_{1}, X_{2}, \ldots, X_{n}$ defined by

$$
X_{i}=\mathbb{P}\left(A \mid \mathcal{G}_{i}\right),
$$

where $1 \leq i \leq n$. As pioneered by Dawid et al. in [6], we refer to such random vectors as coherent.

Definition 1.1. Let $n \geq 2$ be an integer. A family $\left(X_{1}, X_{2}, \ldots, X_{n}\right)$ of random variables on some probability space $(\Omega, \mathcal{F}, \mathbb{P})$ is coherent, if there is a sequence of sub $\sigma$-fields $\mathcal{G}_{1}, \mathcal{G}_{2}, \ldots, \mathcal{G}_{n} \subset \mathcal{F}$ and an event $A \in \mathcal{F}$ such that

$$
X_{i}=\mathbb{E}\left(1_{A} \mid \mathcal{G}_{i}\right), \quad i=1,2, \ldots, n .
$$

*University of Warsaw, Poland. E-mail: S. Cichomski@uw. edu.pl

${ }^{\dagger}$ University of Warsaw, Poland. E-mail: A. 0sekowski@mimuw. edu.pl 
See [6, 7, 8, 12] for applications to statistics, [1] for applications to economy, [3, 4] for purely probabilistic considerations and [2] for philosophical implications. For notational convenience, hereinafter, we write

$$
\left(X_{1}, X_{2}, \ldots, X_{n}\right) \in \mathcal{C}
$$

whenever we want to indicate that the vector $\left(X_{1}, X_{2}, \ldots, X_{n}\right)$ is coherent. Sometimes, in the literature, the joint distribution of $\left(X_{1}, X_{2}, \ldots, X_{n}\right) \in \mathcal{C}$ is also referred to as coherent.

Now, for a fixed $\alpha \in \mathbb{R}_{+}$and $n \geq 2$, consider the number

$$
\sup _{\left(X_{1}, X_{2}, \ldots, X_{n}\right) \in \mathcal{C}} \mathbb{E} \max _{1 \leq i<j \leq n}\left|X_{i}-X_{j}\right|^{\alpha},
$$

where the supremum is taken over all probability spaces $(\Omega, \mathcal{F}, \mathbb{P})$, all events $A \in \mathcal{F}$ and all sub $\sigma$-fields $\mathcal{G}_{1}, \mathcal{G}_{2}, \ldots, \mathcal{G}_{n} \subset \mathcal{F}$. The question about the explicit formula for this supremum can be regarded as the precise, mathematical reformulation of the initial problem, concerning the maximal spread of coherent opinions. The primary goal of this paper is to answer this question for $\alpha=1$.

Theorem 1.2. Under the above notation, we have

$$
\sup _{\left(X_{1}, X_{2}, \ldots, X_{n}\right) \in \mathcal{C}} \mathbb{E} \max _{1 \leq i<j \leq n}\left|X_{i}-X_{j}\right|= \begin{cases}\frac{1}{2} & \text { if } n=2, \\ 2-\sqrt{2} & \text { if } n=3, \\ \frac{7}{2}-2 \sqrt{2} & \text { if } n=4, \\ \frac{n-2}{n-1} & \text { if } n \geq 5 .\end{cases}
$$

Thus, the supremum behaves in a quite surprising manner: we have a few "irregular" terms corresponding to small values of $n$, and then, for $n \geq 5$, it is given by a nice and compact expression. We would like to point out that the result in special case $n=2$ has been already known in the literature. As Burdzy and Pitman showed in [4], this is a consequence of the identity

$$
|X-Y|=2 \cdot \max (X, Y)-X-Y
$$

and the following sharp estimate established in [10].

Theorem 1.3. For all $n \in \mathbb{Z}_{+}$and any $\left(X_{1}, X_{2}, \ldots, X_{n}\right) \in \mathcal{C}$ satisfying $\mathbb{E} X_{i}=p$ for all $i$, we have

$$
\mathbb{E} \max _{1 \leq i \leq n} X_{i} \leq \frac{p(n-p)}{1+p(n-2)} .
$$

A more general result, concerning the calculation of (1.1) still in the special case $n=2$, but for a nontrivial range of the parameters $\alpha$, was established independently in $[1,5]$ with the use of geometric techniques in Hilbert spaces.

Theorem 1.4. For any $\alpha \in(0,2]$ we have

$$
\sup _{(X, Y) \in \mathcal{C}} \mathbb{E}|X-Y|^{\alpha}=2^{-\alpha}
$$

Finally, we would like to mention that our result can also be regarded as a generalization of the martingale diameter problem, see e.g. [9, 11].

In the next section we apply an appropriate symmetrization and reduce the problem of calculating the left-hand side of (1.2) to the analysis of the simpler expression sup $\mathbb{E} \max _{1 \leq i \leq n} X_{i}$, where the supremum is taken over all coherent vectors satisfying 
certain symmetry constraints. Then, in Section 3, using various technical combinatorial arguments, we gradually simplify the context: we show that the extremal coherent vectors (i.e. those for which the simpler supremum is attained) can be assumed to satisfy more and more structural properties. After several steps, this allows us to express the supremum as the extremal value of a certain function of one variable, which in turn can be computed explicitly. Our approach was inspired by the paper [3] by Burdzy and Pal: in that article, a related problem for coherent vectors was also studied with the use of a certain discretization and subsequent combinatorial reductions.

\section{Basic reductions and symmetrizations}

Our starting point is the following discretization, which enables us to restrict our argument to random variables taking values in a finite set. From now on, we will often use the shorter notation and write $X$ instead of $\left(X_{1}, X_{2}, \ldots, X_{n}\right)$.

Proposition 2.1. We have the identity

$$
\sup _{X \in \mathcal{C}} \mathbb{E} \max _{1 \leq i<j \leq n}\left|X_{i}-X_{j}\right|=\sup _{\substack{m \in\{1,2,3, \ldots\} \\ X \in \mathcal{C}(n, m)},} \mathbb{E} \max _{1 \leq i<j \leq n}\left|X_{i}-X_{j}\right|,
$$

where $\mathcal{C}(n, m)$ is the set of all $X=\left(X_{1}, X_{2}, \ldots, X_{n}\right) \in \mathcal{C}$ such that $X_{i}$ takes at most $m$ different values for every $1 \leq i \leq n$.

Proof. See $[3,5]$. We omit the details.

Next, we have the following simple, yet very useful observation.

Lemma 2.2. Let $\left\{G_{1}, G_{2}, \ldots, G_{m}\right\}$ be a finite partition of $\Omega$, let $A \in \mathcal{F}$ be an arbitrary event and put $Y=\mathbb{E}\left(1_{A} \mid \sigma\left(G_{1}, G_{2}, \ldots, G_{m}\right)\right)$. Then for any $y \in(0,1]$ such that $\mathbb{P}(Y=y)>$ 0 , we have

$$
\mathbb{P}\left(\{Y=y\} \cap A^{c}\right)=\mathbb{P}(\{Y=y\} \cap A) \cdot \frac{1-y}{y} .
$$

Proof. For any $G \in \sigma\left(G_{1}, G_{2}, \ldots, G_{m}\right)$ such that $\mathbb{E}\left(1_{A} \mid G\right)=y$, we write

$$
y=\frac{\mathbb{P}(A \cap G)}{\mathbb{P}(G)}=\frac{\mathbb{P}(A \cap G)}{\mathbb{P}(A \cap G)+\mathbb{P}\left(A^{c} \cap G\right)} .
$$

This implies $y \cdot\left(\mathbb{P}(A \cap G)+\mathbb{P}\left(A^{c} \cap G\right)\right)=\mathbb{P}(A \cap G)$ and hence $\mathbb{P}\left(A^{c} \cap G\right)=\frac{1-y}{y} \cdot \mathbb{P}(A \cap G)$. It remains to take $G=\{Y=y\}$; we have $G \in \sigma\left(G_{1}, G_{2}, \ldots, G_{m}\right)$, since $Y$ is measurable with respect to the latter $\sigma$-algebra.

Now we will establish the following reduction.

Proposition 2.3. We have the identity

$$
\sup _{\substack{m \in\{1,2, \ldots\} \\ X \in \mathcal{C}(n, m)}} \mathbb{E} \max _{1 \leq i<j \leq n}\left|X_{i}-X_{j}\right|=\sup _{\substack{m \in\{1,2, \ldots\}, X \in \mathcal{C}^{\prime}(n, m)}} \mathbb{E} \max _{1 \leq i<j \leq n}\left|X_{i}-X_{j}\right|,
$$

where $\mathcal{C}^{\prime}(n, m)$ is the subset of all $X \in \mathcal{C}(n, m)$ which satisfy

$$
\mathbb{P}\left(\left\{\max _{1 \leq i \leq n} X_{i}=1\right\} \cup\left\{\min _{1 \leq j \leq n} X_{j}=0\right\}\right)=1
$$


Proof. Fix $X \in \mathcal{C}(n, m)$. Then there is a probability space $(\Omega, \mathcal{F}, \mathbb{P})$, an event $A \in \mathcal{F}$ and $\sigma$-algebras $\mathcal{G}_{1}, \mathcal{G}_{2}, \ldots, \mathcal{G}_{n}$ such that

$$
X_{i}=\mathbb{E}\left(1_{A} \mid \mathcal{G}_{i}\right), \quad i=1,2, \ldots, n .
$$

With no loss of generality, we may assume that the probability space is non-atomic. Now we will perform a sequence of transformations of the variables $X_{i}$ (or rather of the corresponding $\sigma$-algebras $\mathcal{G}_{i}$ ), after which

- the maximum $\max _{1 \leq i \leq n} X_{i}$ will increase to 1 on $A$;

- the minimum $\min _{1 \leq i \leq n} X_{i}$ will decrease to 0 on $A^{c}$;

- the expectation $\mathbb{E} \max _{1 \leq i<j \leq n}\left|X_{i}-X_{j}\right|$ will increase or stay unchanged.

This will clearly yield the claim. For a given $i \in\{1,2, \ldots, n\}$, the transformation of $X_{i}$ can be described as follows. Split the set $\left\{\max _{1 \leq j \leq n} X_{j}=X_{i}\right\} \cap A$ into the events

$$
A_{i, x}=\left\{\max _{1 \leq j \leq n} X_{j}=X_{i}\right\} \cap A \cap\left\{X_{i}=x\right\}, \quad x \in[0,1] .
$$

Fix $x \in[0,1)$ such that $\mathbb{P}\left(A_{i, x}\right)>0$; there is a finite number of such parameters, since $X_{i}$ takes values in a finite set. Furthermore, we have $x>0$, since $\max _{1 \leq j \leq n} X_{j}>0$ almost surely on $A$. By Lemma 2.2, there exists an event $\tilde{A}_{i, x} \subset\left\{X_{i}=x\right\} \cap A^{c}$ satisfying $\mathbb{P}\left(\tilde{A}_{i, x}\right)=\mathbb{P}\left(A_{i, x}\right) \cdot \frac{1-x}{x}$. We introduce the modification $\tilde{X}_{i}$ of $X_{i}$, given by

$$
\tilde{X}_{i}(\omega)= \begin{cases}1, & \text { for } \omega \in A_{i, x} \\ 0, & \text { for } \omega \in \tilde{A}_{i, x} \\ X_{i}(\omega), & \text { otherwise }\end{cases}
$$

or equivalently, $\tilde{X}_{i}=\mathbb{E}\left(1_{A} \mid \sigma\left(\mathcal{G}_{i}, A_{i, x}, \tilde{A}_{i, x}\right)\right)$. Setting $\tilde{X}_{j} \equiv X_{j}$ for $j \neq i$, we see that

$$
\begin{aligned}
\mathbb{E} \max _{1 \leq k<l \leq n}\left|\tilde{X}_{k}-\tilde{X}_{l}\right|-\mathbb{E} \max _{1 \leq k<l \leq n}\left|X_{k}-X_{l}\right| & \geq \mathbb{P}\left(A_{i, x}\right) \cdot(1-x)-\mathbb{P}\left(\tilde{A}_{i, x}\right) \cdot x \\
& =\mathbb{P}\left(A_{i, x}\right) \cdot\left[(1-x)-\frac{1-x}{x} \cdot x\right]=0
\end{aligned}
$$

and hence the passage $X \mapsto \tilde{X}$ does not decrease the maximized expectation. Furthermore, note that $\max _{1 \leq j \leq n} \tilde{X}_{j}$ has increased to 1 on $A_{i, x}$. The desired transformation of $X_{i}$ is obtained by applying the above modification for all $x \in(0,1)$ with $\mathbb{P}\left(A_{i, x}\right)>0$.

Now, performing the above transformations of $X_{1}, X_{2}, \ldots, X_{n}$, we obtain a new vector $X$ for which

$$
\mathbb{P}\left(\left\{\max _{1 \leq i \leq n} X_{i}=1\right\} \cap A\right)=\mathbb{P}(A) .
$$

Furthermore, applying the above procedure to the coherent sequence $\left(1-X_{1}, 1-\right.$ $X_{2}, \ldots, 1-X_{n}$ ) (corresponding to the event $A^{c}$ ), we may also guarantee that

$$
\mathbb{P}\left(\left\{\min _{1 \leq i \leq n} X_{i}=0\right\} \cap A^{c}\right)=\mathbb{P}\left(A^{c}\right),
$$

which completes the proof.

Remark 2.4. It follows easily from the above argument that if $X \in \mathcal{C}^{\prime}(n, m)$, then the corresponding event $A$ satisfies $A=\left\{\max _{1 \leq i \leq m} X_{i}=1\right\}$ and $A^{c}=\left\{\min _{1 \leq i \leq n} X_{i}=0\right\}$, up to sets of probability zero. 
Our next step is to simplify the maxima in (2.2). Let $X=\left(X_{1}, X_{2}, \ldots, X_{n}\right) \in \mathcal{C}^{\prime}(n, m)$ with $X_{i}=\mathbb{E}\left(1_{A} \mid \mathcal{G}_{i}\right), i=1,2, \ldots, n$, and let $U$ be random variable, independent of $\mathcal{G}_{1}, \mathcal{G}_{2}, \ldots, \mathcal{G}_{n}$ and $A$, having the two-point distribution $\mathbb{P}(U=0)=\mathbb{P}(U=1)=1 / 2$. We may assume the existence of such a random variable, taking the larger, product probability space if necessary. Next, note that

$$
\max _{1 \leq i<j \leq n}\left|X_{i}-X_{j}\right|=\max _{1 \leq i<j \leq n}\left|\left(1-X_{i}\right)-\left(1-X_{j}\right)\right|
$$

almost surely. Thus we can rewrite the right-hand side of (2.2) as

$$
\sup _{\substack{m \in\{1,2, \ldots\},\left(X_{1}, X_{2}, \ldots, X_{n}\right) \in \mathcal{C}^{\prime}(n, m)}} \mathbb{E} \max _{1 \leq i<j \leq n}\left|\tilde{X}_{i}-\tilde{X}_{j}\right|,
$$

where $\tilde{X}$ is a mixture of $X$ and $1-X$, given by

$$
\left(\tilde{X}_{1}, \tilde{X}_{2}, \ldots, \tilde{X}_{n}\right)=U \cdot\left(X_{1}, X_{2}, \ldots, X_{n}\right)+(1-U) \cdot\left(1-X_{1}, 1-X_{2}, \ldots, 1-X_{n}\right) .
$$

Of course, $\tilde{X}$ takes values in a finite set. Furthermore, we have $\mathbb{E} \tilde{X}_{i}=\frac{1}{2}$ for all $i$ and, as the set of coherent distributions on $[0,1]^{n}$ is convex (see [4]), we conclude that $\tilde{X} \in \mathcal{C}$. Let us be more specific here. We take the $\sigma$-algebras $\tilde{\mathcal{G}}_{i}=\sigma\left(\mathcal{G}_{i}, U\right)$ and $\tilde{A}=(A \cap\{U=1\}) \cup\left(A^{c} \cap\{U=0\}\right)$; since $U$ is measurable with respect to $\tilde{\mathcal{G}}_{i}$ and independent of $A$, we indeed obtain

$$
\mathbb{E}\left(1_{\tilde{A}} \mid \tilde{\mathcal{G}}_{i}\right)=1_{\{U=1\}} \mathbb{E}\left(1_{A} \mid \tilde{\mathcal{G}}_{i}\right)+1_{\{U=0\}} \mathbb{E}\left(1_{A^{c}} \mid \tilde{\mathcal{G}}_{i}\right)=U X_{i}+(1-U)\left(1-X_{i}\right)
$$

for all $i$. A similar argument, based on the splitting $\Omega$ into $\Omega \cap\{U=0\}$ and $\Omega \cap\{U=1\}$, shows that

$$
\mathbb{P}\left(\bigcap_{i=1}^{n}\left\{\tilde{X}_{i}=x_{i}\right\} \cap \tilde{A}\right)=\mathbb{P}\left(\bigcap_{i=1}^{n}\left\{\tilde{X}_{i}=1-x_{i}\right\} \cap \tilde{A}^{c}\right),
$$

for any sequence $\left(x_{i}\right)_{i=1}^{n} \subset[0,1]$. See Figure 1 .

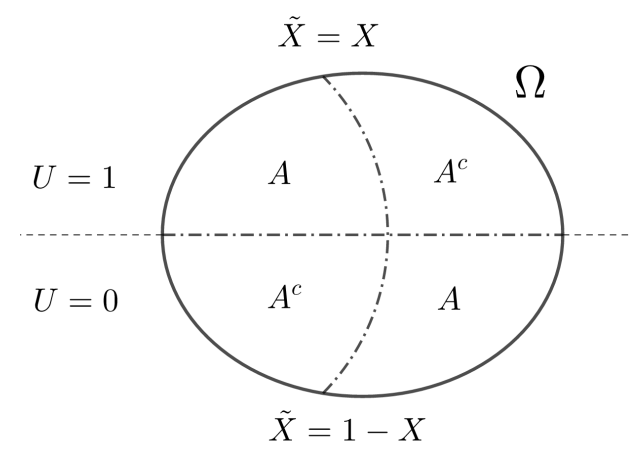

Figure 1: The modified sequence $\tilde{X}$.

As a direct consequence of (2.4) and the above discussion, we obtain the following

Proposition 2.5. For any $n \geq 2$, we have the equality

$$
\sup _{X \in \mathcal{C}} \mathbb{E} \max _{1 \leq i<j \leq n}\left|X_{i}-X_{j}\right|=\sup _{\substack{m \in\{1,2, \ldots\}, X \in \mathcal{C}^{\prime \prime}(n, m)}} \mathbb{E} \max _{1 \leq i<j \leq n}\left|X_{i}-X_{j}\right|
$$

where $\mathcal{C}^{\prime \prime}(n, m)$ is the subset of all those $X \in \mathcal{C}(n, m)$ that satisfy (2.3) and (2.5). 
Remark 2.6. By (2.5), all the coordinates of an arbitrary vector $X \in C^{\prime \prime}(n, m)$ have expectation $1 / 2$; this in particular implies that the event $A$ which "generates" $X$ satisfies $\mathbb{P}(A)=1 / 2$. Actually, (2.5) yields the stronger symmetry property of $X$ around $\frac{1}{2}$ : we have the equality of distributions

$$
\left(X_{i}-\frac{1}{2}\right)_{i=1}^{n} \stackrel{\mathcal{D}}{=}\left(\frac{1}{2}-X_{i}\right)_{i=1}^{n} .
$$

The above remark enables the following further reduction.

Proposition 2.7. For any $n \geq 2$, we have

$$
\sup _{\substack{m \in\{1,2, \ldots\}, X \in \mathcal{C}^{\prime \prime}(n, m)}} \mathbb{E} \max _{1 \leq i<j \leq n}\left|X_{i}-X_{j}\right|+1=2 \cdot \sup _{\substack{m \in\{1,2, \ldots\}, X \in \mathcal{C}^{\prime \prime}(n, m)}} \mathbb{E} \max _{1 \leq i \leq n} X_{i} .
$$

Proof. We may write

$$
\max _{1 \leq i<j \leq n}\left|X_{i}-X_{j}\right|=\max _{1 \leq i, j \leq n}\left(X_{i}-X_{j}\right)=\max _{1 \leq i \leq n}\left(X_{i}-\frac{1}{2}\right)+\max _{1 \leq j \leq n}\left\{-\left(X_{j}-\frac{1}{2}\right)\right\},
$$

and hence by (2.6) we get

$$
\sup _{\substack{m \in\{1,2, \ldots\}, X \in \mathcal{C}^{\prime \prime}(n, m)}} \mathbb{E} \max _{1 \leq i<j \leq n}\left|X_{i}-X_{j}\right|=2 \cdot \sup _{\substack{m \in\{1,2, \ldots\}, X \in \mathcal{C}^{\prime \prime}(n, m)}} \mathbb{E} \max _{1 \leq i \leq n}\left(X_{i}-\frac{1}{2}\right)
$$

which completes the proof.

By the above reductions, we see that it is enough to handle the expression

$$
\sup _{\substack{m \in\{1,2, \ldots\}, X \in \mathcal{C}^{\prime \prime}(n, m)}} \mathbb{E} \max _{1 \leq i \leq n} X_{i}=\sup _{\substack{m \in\{1,2, \ldots\}, X \in \mathcal{C}^{\prime \prime}(n, m)}}\left(\mathbb{E}\left(1_{A} \max _{1 \leq i \leq n} X_{i}\right)+\mathbb{E}\left(1_{A^{c}} \max _{1 \leq i \leq n} X_{i}\right)\right) .
$$

Observe that for a fixed $m$ and an $X \in \mathcal{C}^{\prime \prime}(n, m)$, we have $1_{A} \cdot \max _{1 \leq i \leq n} X_{i}=1_{A}$ almost surely, due to Remark 2.4. In addition, we have the identity

$$
\mathbb{P}\left(\left\{\max _{1 \leq j \leq n} X_{j}=X_{i}\right\} \cap\left\{X_{i}=x\right\} \cap A^{c}\right)=\mathbb{P}\left(\left\{\min _{1 \leq j \leq n} X_{j}=X_{i}\right\} \cap\left\{X_{i}=1-x\right\} \cap A\right),
$$

for $1 \leq i \leq n$ and $x \in[0,1]$, which follows from (2.5). In other words, the distributions of $1_{A^{c}} \max _{1 \leq i \leq n} X_{i}$ and $1_{A}-1_{A} \min _{1 \leq i \leq n} X_{i}$ coincide, and we get the following.

Proposition 2.8. For a given $n \geq 2$, a sequence $X$ is a maximizer of (2.7) if and only if it minimizes the quantity

$$
\inf _{\substack{m \in\{1,2, \ldots\}, X \in \mathcal{C}^{\prime \prime}(n, m)}} \mathbb{E}\left(1_{A} \cdot \min _{1 \leq i \leq n} X_{i}\right) .
$$

The main advantage of this proposition is that it allows us to restrict the analysis of the sequences $X$ to the set $A$.

\section{Combinatorial optimization}

Now we are going to present a combinatorial analysis of (2.8), which will be done by some geometrical considerations in a slightly different setup. Let us start with introducing some auxiliary notation. Throughout, we assume that $n \geq 2$ is a fixed integer. 


\section{Experts' opinions}

Let $(\Omega, \mathcal{F}, \mathbb{P})$ be a non-atomic probability space and let $A$ be a fixed event satisfying $\mathbb{P}(A)=1 / 2$. For an event $S \subset A$ and any $x \in(0,1]$, we set

$$
S^{x}:=S \times\{x\} \quad \subset A \times(0,1] .
$$

In our considerations below, it will be convenient to interpret $S^{x}$ as horizontal line segments at level $x$, or finite unions of sets of this type. We assign to each $S^{x}$ the corresponding function $\mathcal{S}^{x}: A \rightarrow[0,1]$, given by

$$
\mathcal{S}^{x}(\omega)= \begin{cases}x, & \text { for } \omega \in S \\ 1, & \text { for } \omega \in A \backslash S .\end{cases}
$$

For $k \in\{1,2, \ldots\}$, we denote by $\Lambda(n, k)$ the family of all sequences $\left(S_{1}^{x_{1}}, S_{2}^{x_{2}}, \ldots, S_{k}^{x_{k}}\right)$ which satisfy

$$
\sum_{i=1}^{k} \mathbb{P}\left(S_{i}\right)=(n-1) \cdot \frac{1}{2}
$$

and

$$
\sum_{\left\{1 \leq i \leq k: x_{i}=x\right\}} \mathbb{P}\left(S_{i}\right)=\frac{x}{1-x} \cdot \sum_{\left\{1 \leq j \leq k: x_{j}=1-x\right\}} \mathbb{P}\left(S_{j}\right),
$$

for every $x \in(0,1)$. In this setup, we consider the following, new optimization problem:

$$
\inf _{\substack{(\Omega, \mathcal{F}, \mathbb{P}), A \in \mathcal{F}, \mathbb{P}(A)=\frac{1}{2}}} \inf _{\substack{k \in\{1,2, \ldots\},\left(S_{1}^{x_{1}}, S_{2}^{x_{2}}, \ldots, S_{k}^{x_{k}}\right) \in \Lambda(n, k)}} \int_{A} \min \left(\mathcal{S}_{1}^{x_{1}}, \mathcal{S}_{2}^{x_{2}}, \ldots, \mathcal{S}_{k}^{x_{k}}\right) \mathrm{d} \mathbb{P} .
$$

By a straightforward measure-preserving transformation argument, we see that the infimum inside does not depend on the choice of the probability space or the event $A$. In other words, the above quantity is equal to

$$
\inf _{\substack{k \in\{1,2, \ldots\},\left(S_{1}^{x_{1}}, S_{2}^{x_{2}}, \ldots, S_{k}^{x_{k}}\right) \in \Lambda(n, k)}} \int_{A} \min \left(\mathcal{S}_{1}^{x_{1}}, \mathcal{S}_{2}^{x_{2}}, \ldots, \mathcal{S}_{k}^{x_{k}}\right) \mathrm{dP} .
$$

Proposition 3.1. The value of (2.8) is not smaller than the value of (3.3).

Proof. Fix $m \in\{1,2, \ldots\}, X \in \mathcal{C}^{\prime \prime}(n, m)$ and the corresponding "generating" event $A$. As we have seen in Remark 2.6, we have $\mathbb{P}(A)=1 / 2$. For a given $1 \leq i \leq n$, let $\left\{x_{1}^{i}, x_{2}^{i}, \ldots, x_{m}^{i}\right\}$ denote the set of values attained by $X_{i}, 1 \leq i \leq n$ (if $X_{i}$ takes less than $m$ different values, we add some extra, superfluous elements to the set). Introduce the events

$$
T_{i, j}=\left\{\omega \in A: X_{i}(\omega)=x_{j}^{i}\right\},
$$

for $1 \leq i \leq n$ and $1 \leq j \leq m$. Since $\mathbb{P}(A)=1 / 2$, we have the straightforward equality

$$
\sum_{\substack{1 \leq i \leq n, 1 \leq j \leq m}} \mathbb{P}\left(T_{i, j}\right)=n \cdot \frac{1}{2} .
$$

Next, for a fixed $1 \leq i \leq n$ and $x \in(0,1)$, we may also write

$$
\begin{aligned}
\sum_{\left\{1 \leq j \leq m: x_{j}^{i}=x\right\}} \mathbb{P}\left(T_{i, j}\right) & =\frac{x}{1-x} \cdot \sum_{\left\{1 \leq j \leq m: x_{j}^{i}=x\right\}} \mathbb{P}\left(\left\{X_{i}=x\right\} \cap A^{c}\right) \\
& =\frac{x}{1-x} \cdot \sum_{\left\{1 \leq j \leq m: x_{j}^{i}=1-x\right\}} \mathbb{P}\left(T_{i, j}\right),
\end{aligned}
$$


where the first equality is due to the Lemma 2.2 and the second one is a consequence of (2.5). Adding up the above equalities for $1 \leq i \leq n$ yields the condition (3.2) for the family $\left(T_{i, j}^{x_{j}^{i}}\right)_{i, j}$. Next, let $\left(U_{i}\right)_{i=1}^{n}$ be a partition of $A$ such that $U_{i} \in \mathcal{F}$ and $U_{i} \subset\left\{X_{i}=1\right\}$ for all $1 \leq i \leq n$, up to a set of measure zero. The existence of such a partition is an obvious consequence of Remark 2.4. Define a modification $\left(S_{i, j}^{x_{j}^{i}}\right)_{i, j}$ of $\left(T_{i, j}^{x_{j}^{i}}\right)_{i, j}$ by

$$
S_{i, j}= \begin{cases}T_{i, j} \backslash U_{i}, & \text { if } x_{j}^{i}=1 \\ T_{i, j}, & \text { if } x_{j}^{i} \neq 1\end{cases}
$$

Since $\mathbb{P}(A)=1 / 2$, we get

$$
\sum_{\substack{1 \leq i \leq n \\ 1 \leq j \leq m}} \mathrm{P}\left(S_{i, j}\right)=(n-1) \cdot \frac{1}{2}
$$

and the condition (3.2) is still satisfied for $\left(S_{i, j}^{x_{j}^{i}}\right)_{i, j}$ (the sets $T_{i, j}$ are modified only if $x_{j}^{i}=1$, and these values are not considered in (3.2)). Consequently, we have $\left(S_{i, j}^{x_{j}^{i}}\right)_{i, j} \in$ $\bigcup_{k \geq 1} \Lambda(n, k)$ and hence

$$
\mathbb{E}\left(1_{A} \cdot \min _{1 \leq i \leq n} X_{i}\right)=\int_{A} \min _{\substack{1 \leq i \leq n, 1 \leq j \leq m}} \mathcal{S}_{i, j}^{x_{j}^{i}} \mathrm{dP}
$$

is not smaller than the quantity in (3.3). Since $m$ and $X$ were arbitrary, the proof is complete.

Our plan is to solve the problem (3.3), by performing a sequence of combinatorial and geometrical reductions. We start with some simple observations. First, note that by (3.2), a sequence $\left(S_{1}^{x_{1}}, S_{2}^{x_{2}}, \ldots, S_{k}^{x_{k}}\right) \in \Lambda(n, k)$ enjoys a sort of skew-symmetry around $1 / 2$ : in particular, if a level $x$ belongs to $\left\{x_{1}, x_{2}, \ldots, x_{k}\right\}$, then so does $1-x$. Second, obviously, the integral in (3.3) does not depend on the order of the sets $S_{1}^{x_{1}}, S_{2}^{x_{2}}, \ldots, S_{k}^{x_{k}}$, so we may permute them arbitrarily. Furthermore, note that if we split $S_{k}^{x_{k}}$ into two sets $\tilde{S}_{k}^{x_{k}}$ and $\tilde{S}_{k+1}^{x_{k}}$ (of course, the level $x_{k}$ needs to be preserved) and replace $\left(S_{1}^{x_{1}}, S_{2}^{x_{2}}, \ldots, S_{k}^{x_{k}}\right.$ ) with $\left(S_{1}^{x_{1}}, S_{2}^{x_{2}}, \ldots, S_{k-1}^{x_{k-1}}, \tilde{S}_{k}^{x_{k}}, \tilde{S}_{k+1}^{x_{k}}\right)$, then the integral will not change either; a similar phenomenon occurs if we splice two disjoint sets lying at the same level. In other words, given a sequence $\left(S_{1}^{x_{1}}, S_{2}^{x_{2}}, \ldots, S_{k}^{x_{k}}\right)$, we may cut some of the sets into pieces or merge some of them, with no effect on the optimized expression (3.3).

The next step is the following.

Proposition 3.2. The problem (3.3) can be rewritten as

$$
\inf _{\substack{k \in\{1,2, \ldots\},\left(S_{1}^{x_{1}}, S_{2}^{x_{2}}, \ldots, S_{k}^{x_{k}}\right) \in \Lambda^{\star}(n, k)}} \int_{A} \min \left(\mathcal{S}_{1}^{x_{1}}, \mathcal{S}_{2}^{x_{2}}, \ldots, \mathcal{S}_{k}^{x_{k}}\right) \mathrm{d} \mathbb{P}
$$

where $\Lambda^{\star}(n, k)$ is the subset of all those $\left(S_{1}^{x_{1}}, S_{2}^{x_{2}}, \ldots, S_{k}^{x_{k}}\right) \in \Lambda(n, k)$, which satisfy

$$
\mathbb{P}\left(S_{i} \cap S_{j}\right)=0 \quad \text { whenever } \quad x_{i}, x_{j} \leq \frac{1}{2} .
$$

Proof. Fix $k \in\{1,2, \ldots\}$, a vector $\left(S_{1}^{x_{1}}, S_{2}^{x_{2}}, \ldots, S_{k}^{x_{k}}\right) \in \Lambda(n, k)$ and assume that the condition (3.5) is not satisfied for some $0<x_{i} \leq x_{j} \leq \frac{1}{2}$. Of course, the claim will follow if we construct a modification $\left(\tilde{S}_{1}^{x_{1}}, \tilde{S}_{2}^{x_{2}}, \ldots, \tilde{S}_{k}^{x_{k}}\right) \in \Lambda^{\star}(n, k)$ such that

$$
\int_{A} \min \left(\mathcal{S}_{1}^{x_{1}}, \mathcal{S}_{2}^{x_{2}}, \ldots, \mathcal{S}_{k}^{x_{k}}\right) \mathrm{d} \mathbb{P} \geq \int_{A} \min \left(\tilde{\mathcal{S}}_{1}^{x_{1}}, \tilde{\mathcal{S}}_{2}^{x_{2}}, \ldots, \tilde{\mathcal{S}}_{k}^{x_{k}}\right) \mathrm{d} \mathbb{P} .
$$


There are two possible scenarios: we either have

$$
\mathbb{P}\left(\left\{\min \left(\mathcal{S}_{1}^{x_{1}}, \mathcal{S}_{2}^{x_{2}}, \ldots, \mathcal{S}_{k}^{x_{k}}\right)>\frac{1}{2}\right\} \cap A\right)>0
$$

or

$$
\mathbb{P}\left(\left\{\min \left(\mathcal{S}_{1}^{x_{1}}, \mathcal{S}_{2}^{x_{2}}, \ldots, \mathcal{S}_{k}^{x_{k}}\right)>\frac{1}{2}\right\} \cap A\right)=0 .
$$

In the case of (3.7), we can simply cut off a part of $S_{j}$ which is already covered by a smaller value $x_{i}$ and transfer as much of it as possible to the set $\left\{\min \left(\mathcal{S}_{1}^{x_{1}}, \mathcal{S}_{2}^{x_{2}}, \ldots, \mathcal{S}_{k}^{x_{k}}\right)>\frac{1}{2}\right\}$. See Figure 2. Observe that the modification obtained in this way satisfies (3.6). After a finite number of such transformations, we obtain a sequence $\left(S_{1}^{x_{1}}, S_{2}^{x_{2}}, \ldots, S_{k}^{x_{k}}\right)$ for which either (3.5) holds, or we have (3.8).
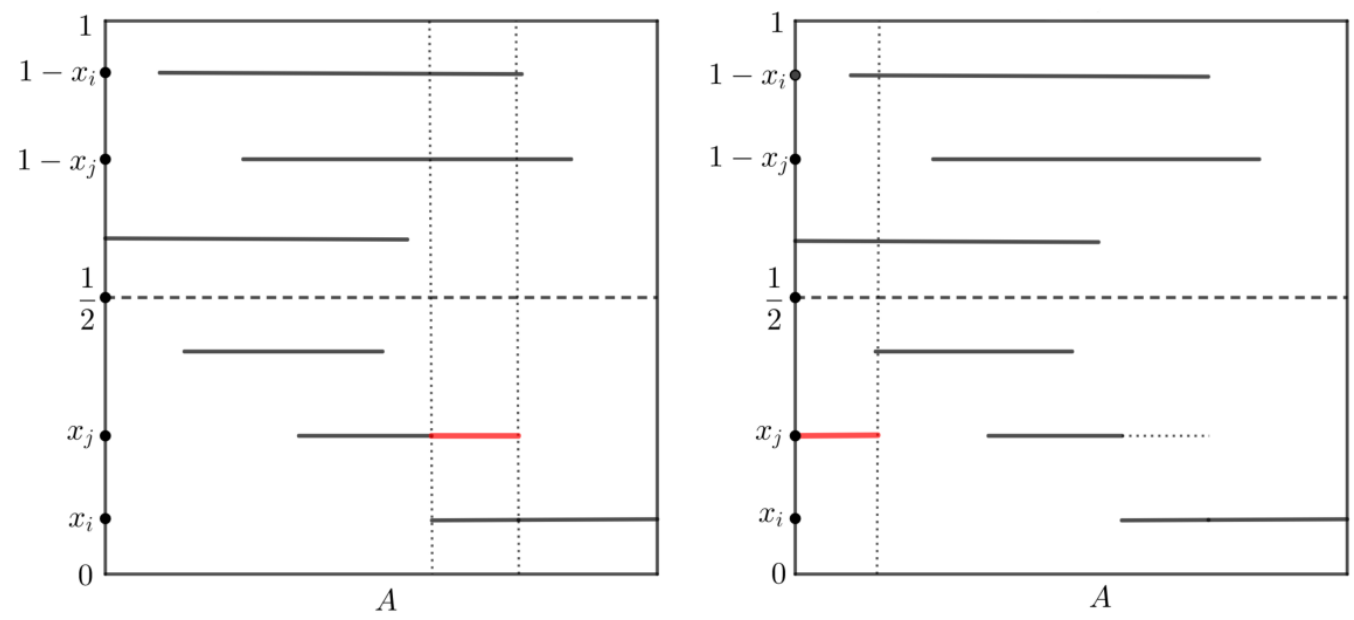

Figure 2: The horizontal line segments are (parts of) graphs of functions $\mathcal{S}_{1}^{x_{1}}, \mathcal{S}_{2}^{x_{2}}, \ldots$, $\mathcal{S}_{n}^{x_{n}}$. In the figure above, the whole problematic intersection (in red) has been moved to the area in which all the other line segments lie above $1 / 2$. However, if the set $\left\{\min \left(\mathcal{S}_{1}^{x_{1}}, \mathcal{S}_{2}^{x_{2}}, \ldots, \mathcal{S}_{k}^{x_{k}}\right)>\frac{1}{2}\right\}$ is too small, it might be impossible to remove the whole problematic intersection - this leads to the scenario (3.8).

Now suppose that (3.5) does not hold for some $0<x_{i} \leq x_{j} \leq \frac{1}{2}$ and we have the equality (3.8). First, set

$$
c=\mathbb{P}\left(S_{i} \cap S_{j}\right), \quad a=\frac{1-x_{i}}{x_{i}} \cdot c \quad \text { and } \quad b=\frac{1-x_{j}}{x_{j}} \cdot c .
$$

Now, let us pick all those $S_{\ell}^{x_{\ell}}$ from the sequence $\left(S_{1}^{x_{1}}, S_{2}^{x_{2}}, \ldots, S_{k}^{x_{k}}\right)$, for which $x_{\ell}=1-x_{i}$ or $x_{\ell}=1-x_{j}$, and arrange them into two lists $\left(S_{i, 1}^{1-x_{i}}, \ldots, S_{i, n_{i}}^{1-x_{i}}\right)$ and $\left(S_{j, 1}^{1-x_{j}}, \ldots, S_{j, n_{j}}^{1-x_{j}}\right)$. Next, we choose two sequences $\left(T_{i, m}\right)_{m=1}^{n_{i}},\left(T_{j, m}\right)_{m=1}^{n_{j}} \subset \mathcal{F}$ such that

and

$$
\begin{array}{lll}
T_{i, m} \subset S_{i, m} & \text { for } & 1 \leq m \leq n_{i}, \\
T_{j, m} \subset S_{j, m} & \text { for } & 1 \leq m \leq n_{j},
\end{array}
$$

$$
\sum_{m=1}^{n_{i}} \mathbb{P}\left(T_{i, m}\right)=a, \quad \sum_{m=1}^{n_{j}} \mathbb{P}\left(T_{j, m}\right)=b .
$$


By (3.2), such $\left(T_{i, m}\right)_{m},\left(T_{j, m}\right)_{m}$ exist (since we assumed that the probability space is nonatomic). Assume further that $x_{0}$ is a number satisfying

$$
\frac{1-x_{0}}{x_{0}} \cdot c=a+b+c,
$$

and observe that $x_{0}<x_{i}$ : indeed, we have $a=\left(1-x_{i}\right) c / x_{i}<a+b+c$. We shall now perform the following transformation:

1. remove $\left(T_{i, m}^{1-x_{i}}\right)_{m=1}^{n_{i}},\left(T_{j, m}^{1-x_{j}}\right)_{m=1}^{n_{j}},\left(S_{i} \cap S_{j}\right)^{x_{i}}$ and $\left(S_{i} \cap S_{j}\right)^{x_{j}}$,

2. add $\left(T_{i, m}^{1-x_{0}}\right)_{m=1}^{n_{i}},\left(T_{j, m}^{1-x_{0}}\right)_{m=1}^{n_{j}},\left(S_{i} \cap S_{j}\right)^{1-x_{0}}$ and $\left(S_{i} \cap S_{j}\right)^{x_{0}}$,

- see Figure 3. It is straightforward to check that the new, modified sequence $\left(S_{\ell}^{x_{\ell}}\right)$ satis-
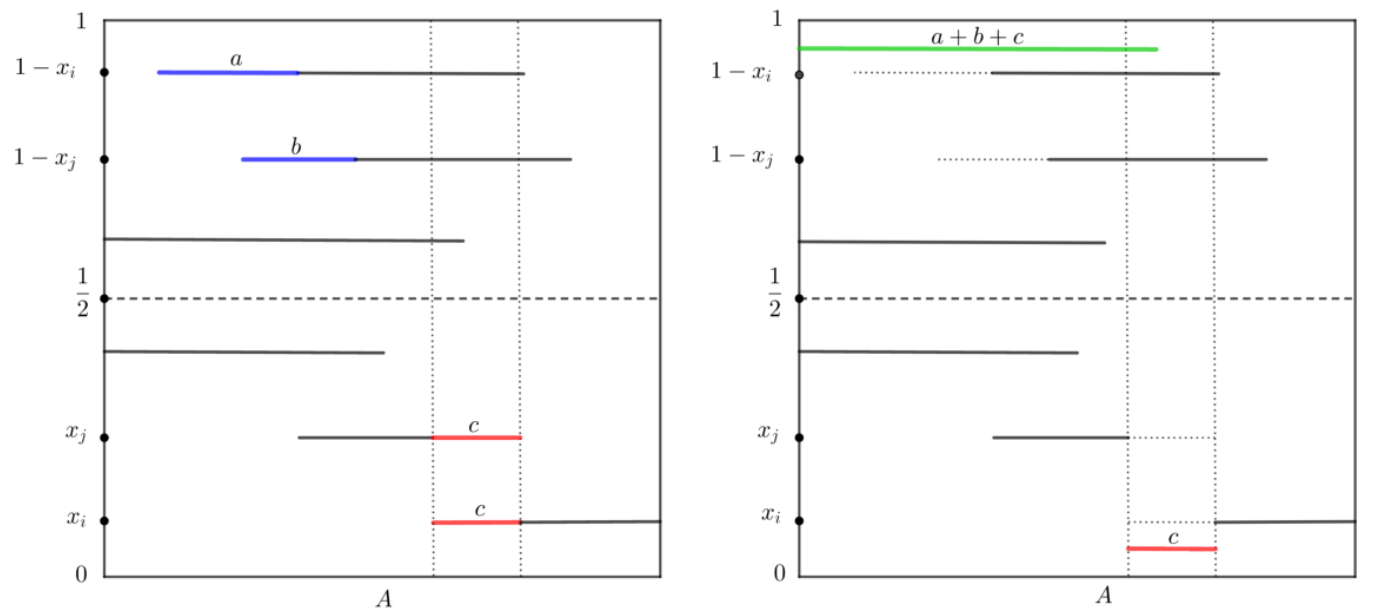

Figure 3: The transformation in the case (3.8). The integral $\int_{A} \min \left(\mathcal{S}_{i}^{x_{i}}\right) \mathrm{dP}$ can only decrease. Indeed, the new line segment of length $c$ on the right picture lies below $x_{i}$; furthermore, though the line segment of length $a+b+c$ might lie on a higher level, the condition (3.8) guarantees that there must be a "layer" of line segments lying below it.

fies (3.1), (3.2) and (3.6). After a finite number of such transformations, we guarantee the validity of (3.5).

Therefore, from now on, we may restrict our analysis of (3.3) to the class $\bigcup_{k \geq 1} \Lambda^{\star}(n, k)$. To proceed, consider a vector

$$
\left(S_{1}^{x_{1}}, S_{2}^{x_{2}}, \ldots, S_{k}^{x_{k}}\right) \in \Lambda^{\star}(n, k)
$$

and introduce a partition of $A$ into the following four basic components. Namely, we first put

$$
A_{1}=\left\{\omega \in A: \min _{1 \leq i \leq k} \mathcal{S}_{i}^{x_{i}}=\frac{1}{2}\right\} \quad \text { and } \quad A_{2}=\left\{\omega \in A: \min _{1 \leq i \leq k} \mathcal{S}_{i}^{x_{i}}>\frac{1}{2}\right\} .
$$

Let $y_{1}, y_{2}, \ldots, y_{m}$ be the collection of values taken by $\min _{1 \leq i \leq k} \mathcal{S}_{i}^{x_{i}}$ on the set $A_{2}$. For $1 \leq j \leq m$, we set

$$
M_{y_{j}}=A_{2} \cap\left\{\min _{1 \leq i \leq k} \mathcal{S}_{i}^{x_{i}}=y_{j}\right\}
$$




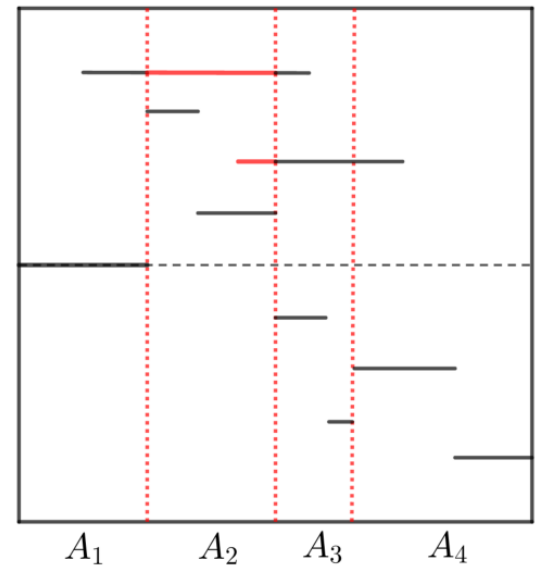

a)

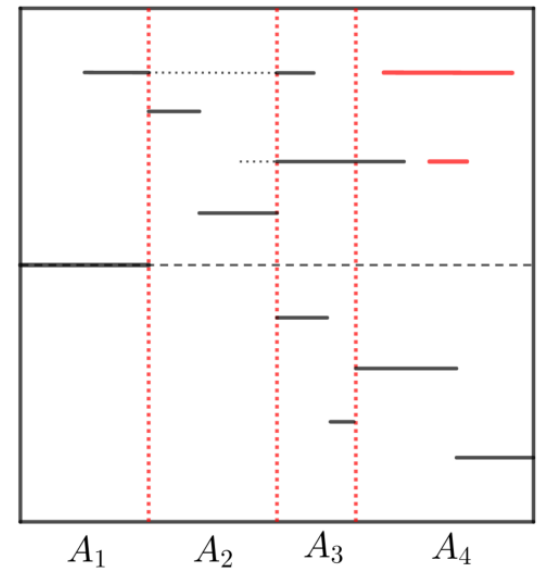

b)

Figure 4: basic components of $A$ : in a) before, in b) after the rearrangement.

By (3.2), we can find events $\left(N_{1-y_{j}}\right)_{j=1}^{m}$ satisfying

$$
N_{1-y_{j}} \subset A \cap\left\{\min _{1 \leq i \leq k} \mathcal{S}_{i}^{x_{i}}=1-y_{j}\right\} \quad \text { and } \quad \mathbb{P}\left(N_{1-y_{j}}\right)=\frac{1-y_{j}}{y_{j}} \cdot \mathbb{P}\left(M_{y_{j}}\right) .
$$

Observe that $\left(N_{1-y_{j}}\right)_{j=1}^{m}$ are disjoint by virtue of Proposition 3.2. Lastly, we define

$$
A_{3}=\bigcup_{1 \leq j \leq m} N_{1-y_{j}} \quad \text { and } \quad A_{4}=\left\{\omega \in A: \min _{1 \leq i \leq k} \mathcal{S}_{i}^{x_{i}}<\frac{1}{2}\right\} \backslash A_{3},
$$

- see Figure 4.a. We can think about the set $A_{3}$ as being generated or induced by $A_{2}$.

Proposition 3.3. The problem (3.4) can be rewritten as

$$
\inf _{\substack{k \in\{1,2, \ldots\},\left(S_{1}^{x_{1}}, S_{2}^{x_{2}}, \ldots, S_{k}^{x_{k}}\right) \in \Lambda^{\star \star}(n, k)}} \int_{A} \min \left(\mathcal{S}_{1}^{x_{1}}, \mathcal{S}_{2}^{x_{2}}, \ldots, \mathcal{S}_{k}^{x_{k}}\right) \mathrm{d} \mathbb{P},
$$

where $\Lambda^{\star \star}(n, k)$ is the subset of all $\left(S_{1}^{x_{1}}, S_{2}^{x_{2}}, \ldots, S_{k}^{x_{k}}\right) \in \Lambda^{\star}(n, k)$ which satisfy

$$
\mathbb{P}\left(A_{2} \cap \bigcup_{1 \leq i<j \leq k}\left(S_{i} \cap S_{j}\right)\right)=0
$$

Proof. Fix $k \in\{1,2, \ldots\}$, a sequence $\left(S_{1}^{x_{1}}, S_{2}^{x_{2}}, \ldots, S_{k}^{x_{k}}\right) \in \Lambda^{\star}(n, k)$ and assume that the condition (3.10) is not satisfied. This in particular implies $\mathbb{P}\left(A_{2}\right)>0$ and hence we must also have $\mathbb{P}\left(A_{3} \cup A_{4}\right)>0$ (see the skew-symmetry of $\left(S_{1}^{x_{1}}, S_{2}^{x_{2}}, \ldots, S_{k}^{x_{k}}\right)$, mentioned above Proposition 3.2). Now, let us fix any $i \in\{1,2, \ldots, k\}$ with $x_{i} \geq \frac{1}{2}$. Then, for any event $T \subset S_{i} \cap A_{2}$ satisfying

$$
T \subset\left\{\min _{1 \leq j \leq k} \mathcal{S}_{j}^{x_{j}}<x_{i}\right\} \quad \text { or } \quad T \subset\left(S_{i} \cap \bigcup_{\substack{1 \leq j \leq k, 1 \\ j \neq i, x_{j}=x_{i}}} S_{j}\right),
$$

we perform the following rearrangement: 
1. remove $T^{x_{i}}$,

2. add a sequence $\left(T_{m}^{x_{i}}\right)_{m=1}^{N}$ of events such that

$$
T_{m} \subset A_{3} \cup A_{4} \quad \text { for } \quad 1 \leq m \leq N,
$$

and

$$
\sum_{m=1}^{N} \mathbb{P}\left(T_{m}\right)=\mathbb{P}(T)
$$

- see Figure $4 \mathrm{~b})$. The existence of $\left(T_{m}^{x_{i}}\right)_{m=1}^{N}$ follows trivially from the condition $\mathbb{P}\left(A_{3} \cup\right.$ $\left.A_{4}\right)>0$ : we allow the overlapping of the sets. The obtained modified sequence belongs to $\Lambda^{\star}(n, \ell)$ for some $\ell$ and the minimum of the corresponding functions is unchanged almost surely on $A$, in comparison to the initial minimum $\min \mathcal{S}_{\ell}^{x_{\ell}}$. It remains to observe that we may guarantee the validity of (3.10), by performing sufficiently many such transformations.

Proposition 3.4. In the problem (3.9), we are allowed to restrict ourselves to those vectors $\left(S_{1}^{x_{1}}, \ldots, S_{k}^{x_{k}}\right) \in \Lambda^{\star \star}(n, k)$, which additionally satisfy

$$
\mathbb{P}\left(A_{2} \cup A_{3}\right)=0,
$$

and

$$
\left|\left\{1 \leq i \leq k: x_{i}>\frac{1}{2}\right\}\right| \leq 1 \text {. }
$$

Proof. The argument follows the above pattern. Namely, we fix $k \in\{1,2, \ldots\}$, a sequence $\left(S_{1}^{x_{1}}, \ldots, S_{k}^{x_{k}}\right) \in \Lambda^{\star \star}(n, k)$ and assume that the condition (3.11) is not satisfied. Let $x>\frac{1}{2}$ be such that $\mathrm{P}\left(M_{x}\right)>0$, where, as before,

$$
M_{x}=A_{2} \cap\left\{\min _{1 \leq i \leq k} \mathcal{S}_{i}^{x_{i}}=x\right\} .
$$

Moreover, recall that $N_{1-x}$ is an event satisfying

$$
N_{1-x} \subset A_{3} \cap\left\{\min _{1 \leq i \leq k} \mathcal{S}_{i}^{x_{i}}=1-x\right\} \quad \text { and } \quad \mathbb{P}\left(N_{1-x}\right)=\frac{1-x}{x} \cdot \mathbb{P}\left(M_{x}\right)
$$

Note that we have

$$
\begin{aligned}
\int_{M_{x} \cup N_{1-x}} \min \left(\mathcal{S}_{1}^{x_{1}}, \mathcal{S}_{2}^{x_{2}}, \ldots, \mathcal{S}_{k}^{x_{k}}\right) \mathrm{d} \mathbb{P} & =x \cdot \mathbb{P}\left(M_{x}\right)+(1-x) \cdot \mathbb{P}\left(N_{1-x}\right) \\
& =\mathbb{P}\left(M_{x} \cup N_{1-x}\right) \cdot\left(x^{2}+(1-x)^{2}\right) \\
& >\mathbb{P}\left(M_{x} \cup N_{1-x}\right) \cdot \frac{1}{2} .
\end{aligned}
$$

Consider the following operation:

1. remove $M_{x}^{x}$ and $N_{1-x}^{1-x}$,

2. add $M_{x}^{\frac{1}{2}}$ and $N_{1-x}^{\frac{1}{2}}$,

- see Figure 5.a. By (3.13), such a transformation yields a sequence from the class $\bigcup_{\ell \in\{1,2, \ldots\}} \Lambda^{\star \star}(n, \ell)$, for which the integral in (3.9) is decreased. After a finite number of 


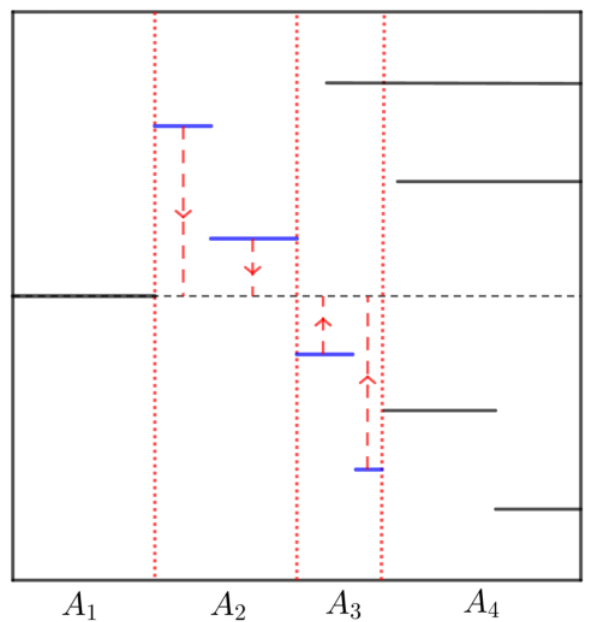

a)

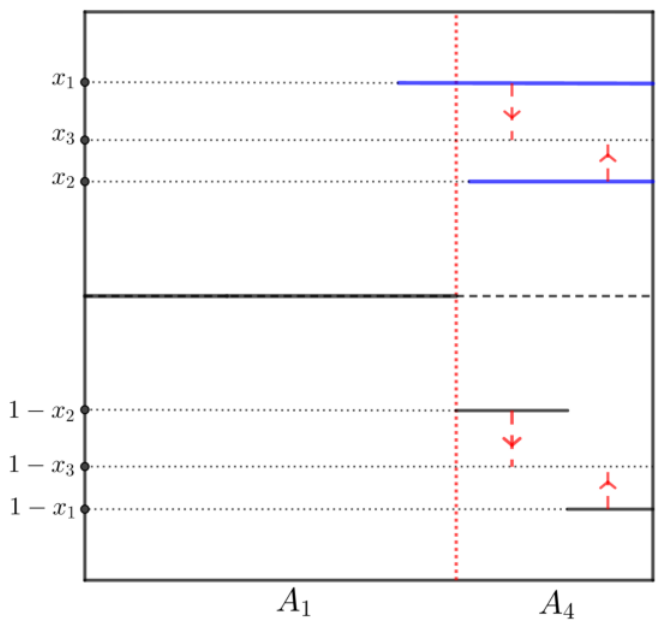

b)

Figure 5: a: removing $A_{2} \cup A_{3}$. b: leveling $x_{1}, x_{2}>\frac{1}{2}, x_{1} \neq x_{2}$.

such operations, we remove $A_{2}$ and thus enforce the validity of (3.11). To guarantee the second condition, we proceed as previously. We start with an arbitrary sequence

$$
\left(S_{1}^{x_{1}}, S_{2}^{x_{2}}, \ldots, S_{k}^{x_{k}}\right) \in \Lambda^{\star \star}(n, k),
$$

for which (3.11) is satisfied, but (3.12) is not. We may assume, performing a permutation of the indices if necessary, that $x_{1}, x_{2}>\frac{1}{2}$ and $s_{1}:=\mathbb{P}\left(S_{1}\right)>0, s_{2}:=\mathbb{P}\left(S_{2}\right)>0$. By (3.2), we can find $T_{1-x_{1}}, T_{1-x_{2}} \in \mathcal{F}$ such that

$$
T_{1-x_{1}} \subset\left\{\min _{1 \leq j \leq k} \mathcal{S}_{j}^{x_{j}}=1-x_{1}\right\}, \quad T_{1-x_{2}} \subset\left\{\min _{1 \leq j \leq k} \mathcal{S}_{j}^{x_{j}}=1-x_{2}\right\},
$$

and

$$
t_{1}:=\mathbb{P}\left(T_{1-x_{1}}\right)=\frac{1-x_{1}}{x_{1}} \cdot s_{1}, \quad t_{2}:=\mathbb{P}\left(T_{1-x_{2}}\right)=\frac{1-x_{2}}{x_{2}} \cdot s_{2} .
$$

Consider the auxiliary equation

$$
t_{1} \cdot \frac{x_{1}}{1-x_{1}}+t_{2} \cdot \frac{x_{2}}{1-x_{2}}=\left(t_{1}+t_{2}\right) \cdot \frac{x_{0}}{1-x_{0}},
$$

that is, equivalently,

$$
1-x_{0}=\frac{t_{1}+t_{2}}{\frac{t_{1}}{1-x_{1}}+\frac{t_{2}}{1-x_{2}}} .
$$

(if $x_{1}$ or $x_{2}$ is equal to 1 , we understand this equation as $x_{0}=1$ ). We will check that

$$
\left(t_{1}+t_{2}\right) \cdot\left(1-x_{0}\right) \leq t_{1} \cdot\left(1-x_{1}\right)+t_{2} \cdot\left(1-x_{2}\right) .
$$

This is obvious for $x_{0}=1$, for the remaining $x_{3}$ we substitute the previous identity and rewrite the estimate in the form

$$
\frac{\left(t_{1}+t_{2}\right)^{2}}{\frac{t_{1}}{1-x_{1}}+\frac{t_{2}}{1-x_{2}}} \leq t_{1} \cdot\left(1-x_{1}\right)+t_{2} \cdot\left(1-x_{2}\right),
$$

or

$$
2 \leq \frac{1-x_{1}}{1-x_{2}}+\frac{1-x_{2}}{1-x_{1}}
$$

which is evident. Having that in mind, let us consider the transformation: 


\section{Experts' opinions}

1. remove $S_{1}^{x_{1}}, S_{2}^{x_{2}}$ and $T_{1-x_{1}}^{1-x_{1}}, T_{1-x_{2}}^{1-x_{2}}$,

2. add $S_{1}^{x_{0}}, S_{2}^{x_{0}}$, and $T_{1-x_{1}}^{1-x_{0}}, T_{1-x_{2}}^{1-x_{0}}$.

By (3.14), the obtained new sequence still belongs to $\bigcup_{\ell>1} \Lambda^{\star \star}(n, \ell)$ and enjoys (3.11). Furthermore, by (3.15), the appropriate minimized integral over $A$ does not increase. It remains to note that after a finite number of the above transformations, the condition (3.12) will become true.

We are almost ready for the proof of our main result. Introduce the auxiliary constant

$$
s(n):=1-2 \inf _{\substack{k \in\{1,2, \ldots\},\left(S_{1}^{x_{1}}, S_{2}^{x_{2}}, \ldots, S_{k}^{x_{k}}\right) \in \Lambda(n, k)}} \int_{A} \min \left(\mathcal{S}_{1}^{x_{1}}, \mathcal{S}_{2}^{x_{2}}, \ldots, \mathcal{S}_{k}^{x_{k}}\right) \mathrm{d} \mathbb{P} .
$$

Theorem 3.5. For any $n$, the number $s(n)$ is equal to the right-hand side of (1.2).

Proof. By the above reductions, in the definition of $s(n)$ we may restrict ourselves to those $\left(S_{1}^{x_{1}}, S_{2}^{x_{2}}, \ldots, S_{k}^{x_{k}}\right) \in \Lambda^{\star \star}(n, k)$, which additionally satisfy (3.11) and (3.12). This is a very simple context: there are at most three different levels of the sets $S_{j}$. See Figure 6. Put $p:=\mathbb{P}\left(A_{1}\right)$ and suppose that the maximal level is equal to $x_{1}$. Note that

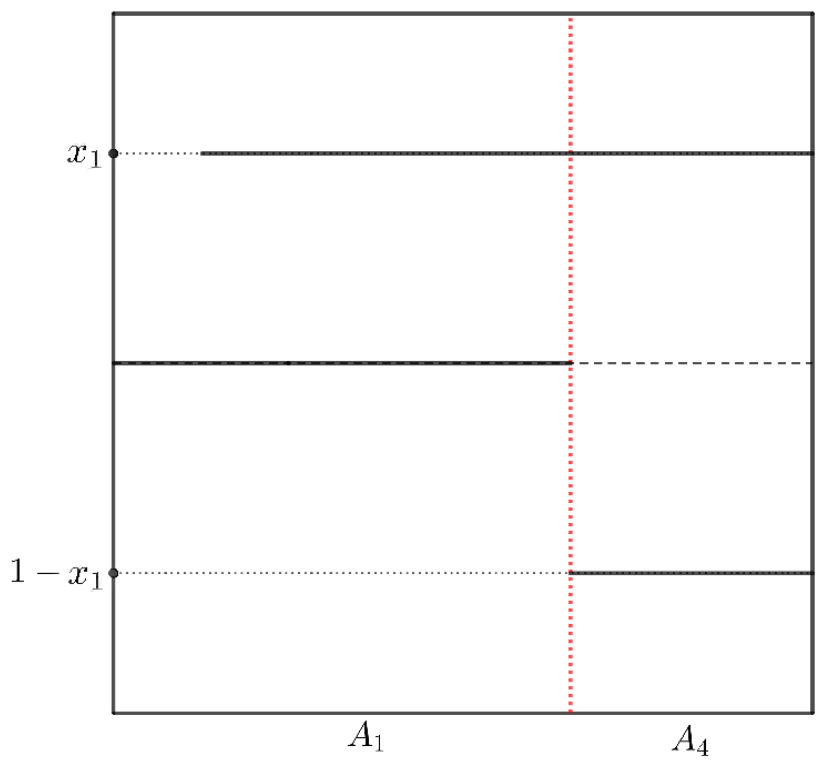

Figure 6: There are at most three different values in the set $\left\{x_{1}, x_{2}, \ldots, x_{k}\right\}$; we may assume that $x_{1}$ is the largest of them.

the random variable $\min \left(\mathcal{S}_{1}^{x_{1}}, \mathcal{S}_{2}^{x_{2}}, \ldots, \mathcal{S}_{k}^{x_{k}}\right) 1_{A}$ is equal to $1 / 2$ on $A_{1}$ and to $1-x_{1}$ on $A_{4}$, so we have

$$
s(n)=1-2 \cdot \inf _{p \in\left[0, \frac{1}{2}\right]}\left[p \cdot \frac{1}{2}+\left(\frac{1}{2}-p\right) \cdot\left(1-x_{1}\right)\right] .
$$

To express $x_{1}$ in terms of $p$, we apply (3.1) and (3.2) with $x=x_{1}$ to obtain

$$
\left[\frac{n-1}{2}-p-\left(\frac{1}{2}-p\right)\right] \cdot \frac{1-x_{1}}{x_{1}}=\frac{1}{2}-p
$$


If $n=2$, we get $p=1 / 2$ and $s(n)=1 / 2$. For $n \geq 3$, the equation (3.16) yields

$$
x_{1}=x_{1}(p)=\frac{n-2}{n-1-2 p}
$$

and hence we obtain

$$
s(n)=1-2 \cdot \inf _{p \in\left[0, \frac{1}{2}\right]}\left[p \cdot \frac{1}{2}+\left(\frac{1}{2}-p\right) \cdot \frac{1-2 p}{n-1-2 p}\right] .
$$

Now we consider the cases $n \in\{3,4\}$ and $n \geq 5$ separately. In the first case, we make some elementary calculations to obtain

$$
\underset{p \in\left[0, \frac{1}{2}\right]}{\operatorname{argmin}}\left[p \cdot \frac{1}{2}+\left(\frac{1}{2}-p\right) \cdot \frac{1-2 p}{n-1-2 p}\right]= \begin{cases}1-\frac{1}{\sqrt{2}}, & \text { for } n=3, \\ \frac{3}{2}-\sqrt{2}, & \text { for } n=4\end{cases}
$$

This gives $s(3)=2-\sqrt{2}$ and $s(4)=\frac{7}{2}-2 \sqrt{2}$, as announced above. For $n \geq 5$, it is straightforward to check the function

$$
t(n, p):=\left[p \cdot \frac{1}{2}+\left(\frac{1}{2}-p\right) \cdot \frac{1-2 p}{n-1-2 p}\right]
$$

satisfies $\partial t(n, p) / \partial p>0$ for $p \in(0,1 / 2)$. Consequently, we get

$$
s(n)=1-2 t(n, 0)=\frac{n-2}{n-1}, \quad \text { for } \quad n \geq 5,
$$

and the proof is complete.

We turn the attention to our main result.

Proof of Theorem 1.2. We need to show that

$$
\sup _{\left(X_{1}, X_{2}, \ldots, X_{n}\right) \in \mathcal{C}} \mathbb{E} \max _{1 \leq i<j \leq n}\left|X_{i}-X_{j}\right|=s(n) .
$$

By Propositions 2.7, 2.8 and 3.1, we get

$$
\sup _{\left(X_{1}, \ldots, X_{n}\right) \in \mathcal{C}} \mathbb{E} \max _{1 \leq i<j \leq n}\left|X_{i}-X_{j}\right| \leq s(n),
$$

and it is enough to check that those bounds cannot be improved. To this end, we construct an appropriate coherent vector $\left(X_{1}, X_{2}, \ldots, X_{n}\right)$ on $([0,1], \mathcal{B}(0,1) ;|\cdot|)$, where $\mathcal{B}(0,1)$ is the $\sigma$-algebra of Borel subsets of $[0,1]$ and $|\cdot|$ is the Lebesgue measure. Fix $p \in(0,1 / 2]$, put $A=[0,1 / 2], B=(1 / 2,1]$ and for $1 \leq i \leq n-1$, consider the families of intervals

$$
A_{1}^{i}=\left(\frac{p(i-1)}{n-1}, \frac{p i}{n-1}\right], \quad A_{2}^{i}=\left(p+\frac{\left(\frac{1}{2}-p\right)(i-1)}{n-1}, p+\frac{\left(\frac{1}{2}-p\right) i}{n-1}\right]
$$

and

$$
B_{1}^{i}=\left(\frac{1}{2}+\frac{p(i-1)}{n-1}, \frac{1}{2}+\frac{p i}{n-1}\right], \quad B_{2}^{i}=\left(\frac{1}{2}+p+\frac{\left(\frac{1}{2}-p\right)(i-1)}{n-1}, \frac{1}{2}+p+\frac{\left(\frac{1}{2}-p\right) i}{n-1}\right] .
$$

It is easy to check that all these intervals are pairwise disjoint and $A_{1}^{i}, A_{2}^{i} \subset A, B_{1}^{i}, B_{2}^{i} \subset$ $B$ for all $i$. Therefore, if we take

$$
\mathcal{G}_{i}=\sigma\left(A_{1}^{i} \cup B_{1}^{i}, A_{2}^{i} \cup\left(B \backslash\left(B_{1}^{i} \cup B_{2}^{i}\right)\right),\left(A \backslash\left(A_{1}^{i} \cup A_{2}^{i}\right)\right) \cup B_{2}^{i}\right)
$$


and $X_{i}=\mathbb{E}\left(1_{A} \mid \mathcal{G}_{i}\right)$, then some straightforward calculations show that

$$
X_{i}= \begin{cases}\frac{1}{2} & \text { on } A_{1}^{i} \cup B_{1}^{i}, \\ 1-x_{1}(p) & \text { on } A_{2}^{i} \cup\left(B \backslash\left(B_{1}^{i} \cup B_{2}^{i}\right)\right), \\ x_{1}(p) & \text { on }\left(A \backslash\left(A_{1}^{i} \cup A_{2}^{i}\right)\right) \cup B_{2}^{i}\end{cases}
$$

for all $1 \leq i \leq n-1$, where $x_{1}(p)$ is given by (3.17). We add a variable $X_{n}=1_{A}$ (which corresponds to taking $\left.\mathcal{G}_{n}=\sigma(A)\right)$. Then the vector $\left(X_{1}, X_{2}, \ldots, X_{n}\right)$ is coherent. For any $\omega \in(0, p]$, we have $X_{n}(\omega)=1$ and, by (3.19), there is an index $i$ such that $X_{i}(\omega)=1 / 2$; consequently,

$$
\mathbb{E} \max _{1 \leq i<j \leq n}\left|X_{i}-X_{j}\right| 1_{[0, p]} \geq \frac{1}{2} p
$$

Similarly, we have

$$
\begin{aligned}
\mathbb{E} \max _{1 \leq i<j \leq n}\left|X_{i}-X_{j}\right| 1_{(p, 1 / 2]} & \geq\left(\frac{1}{2}-p\right) x_{1}(p), \\
\mathbb{E} \max _{1 \leq i<j \leq n}\left|X_{i}-X_{j}\right| 1_{(1 / 2,1 / 2+p]} & \geq \frac{1}{2} p \\
\mathbb{E} \max _{1 \leq i<j \leq n}\left|X_{i}-X_{j}\right| 1_{(1 / 2+p, 1]} & \geq\left(\frac{1}{2}-p\right) x_{1}(p)
\end{aligned}
$$

and hence, summing the above four inequalities, we get

$$
\mathbb{E} \max _{1 \leq i<j \leq n}\left|X_{i}-X_{j}\right| \geq 1-p-(1-2 p)\left(1-x_{1}(p)\right) .
$$

It remains to observe that the supremum of the right-hand side over $p \in(0,1 / 2]$ is precisely $s(n)$ : see (3.18). This completes the proof.

\section{References}

[1] I. Arieli, Y. Babichenko, F. Sandomirskiy, and O. Tamuz, Feasible joint posterior beliefs, 2020, Preprint available at arXiv:2002.11362 [econ.TH].

[2] K. Burdzy, Resonance: From probability to epistemology and back, Imperial College Press, London, 2016. MR-3468703

[3] K. Burdzy and S. Pal, Can coherent predictions be contradictory?, Advances in Applied Probability 53 (2021), 133-161. MR-4232752

[4] K. Burdzy and J. Pitman, Bounds on the probability of radically different opinions, Electron. Commun. Probab. 25 (2020), Paper No. 14, 12 pp. MR-4069734

[5] S. Cichomski, Maximal spread of coherent distributions: a geometric and combinatorial perspective, Master's thesis, University of Warsaw, 2020, available at arXiv:2007.08022 [math.PR].

[6] A. R. Dawid, M. H. DeGroot, and J. Mortera, Coherent combination of experts' opinions, Test 4 (1995), 263-313. MR-1379793

[7] M. H. DeGroot, A bayesian view of assessing uncertainty and comparing expert opinion, Journal of Statistical Planning and Inference 20, 295-306. MR-0976182

[8] M. H. Degroot and J. Mortera, Optimal linear opinion pools, Management Science 37 (1991).

[9] L. E. Dubins, D. Gilat, and I. Meilijson, On the expected diameter of an L2-bounded martingale, The Annals of Probability 37 (2009), 393-402. MR-2489169

[10] L. E. Dubins and J. Pitman, A maximal inequality for skew fields, Zeitschrift für Wahrscheinlichkeitstheorie und Verwandte Gebiete 52 (1980), 219-227. MR-0576883 


\section{Experts' opinions}

[11] A. Osękowski, Estimates for the diameter of a martingale, Stochastics: An International Journal of Probability and Stochastic Processes 87 (2015), 235-256. MR-3316810

[12] R. Ranjan and T. Gneiting, Combining probability forecasts, Journal of the Royal Statistical Society Series B 72 (2010), 71-91. MR-2751244

Acknowledgments. The authors would like to thank an anonymous Referee for the very careful reading of the first version of the paper, for many helpful comments and suggestions. 


\section{Electronic Journal of Probability Electronic Communications in Probability}

\section{Advantages of publishing in EJP-ECP}

- Very high standards

- Free for authors, free for readers

- Quick publication (no backlog)

- Secure publication $\left(\mathrm{LOCKSS}^{1}\right)$

- Easy interface (EJMS²)

\section{Economical model of EJP-ECP}

- Non profit, sponsored by $\mathrm{IMS}^{3}, \mathrm{BS}^{4}$, ProjectEuclid ${ }^{5}$

- Purely electronic

\section{Help keep the journal free and vigorous}

- Donate to the IMS open access fund ${ }^{6}$ (click here to donate!)

- Submit your best articles to EJP-ECP

- Choose EJP-ECP over for-profit journals

\footnotetext{
${ }^{1}$ LOCKSS: Lots of Copies Keep Stuff Safe http://www. lockss.org/

${ }^{2}$ EJMS: Electronic Journal Management System http://www.vtex.lt/en/ejms.html

${ }^{3}$ IMS: Institute of Mathematical Statistics http://www.imstat.org/

${ }^{4}$ BS: Bernoulli Society http://www. bernoulli-society.org/

${ }^{5}$ Project Euclid: https://projecteuclid.org/

${ }^{6}$ IMS Open Access Fund: http://www.imstat.org/publications/open.htm
} 